\title{
Planetary Geologic Mapping
}

\section{A White Paper to \\ The National Research Council \\ 2023 - 2032 Planetary Science and Astrobiology Decadal Survey}

Lead Author:

Peter Mouginis-Mark* (University of Hawaii) pmm@ @higp.hawain.edu Tel: (808) 956-6490

Coauthors (in alphabetical order):

Devon Burr (Northern Arizona Univ.), Devon.Burr@nau.edu

Paul Byrne (North Carolina State Univ.),paul.byrne@nosu.edu

Ken Coles (Indiana Univ. Pennsylvania), kcoles@iup.edu

David A. Crown (Planetary Science Institute), crown@psi.edu Alex Patthoff* (Planetary Science Institute), apatthoff@psi.edu Michael S.Phillips (Univ. Tennessee, Knoxville), mphill58@vols.utk.edu Louise Prockter ${ }^{\star}$ (Johns Hopkins Univ.IAPL), Louise.Prockter@jhuapl.edu Jani Radebaugh* (Brigham Young Univ.), MAPSIT Chair, janirad@byu.edu David A. Williams* (Arizona State University), David.Williams@asu.edu James R. Zimbelman (NASM, Smithsonian Institution), zimbelmani@si.edu

\section{Co-Signer:}

James A. Skinner, Jr. (U.S. Geological Survey), iskinner@usgs.gov

* Member, NASA's Mapping and Planetary Spatial Infrastructure Team (MAPSIT) 


\begin{abstract}
Planetary geologic mapping is a fundamental tool for geologic investigations of planetary surfaces; geoscience maps provide important context for establishing and comparing broadly occurring geologic units, as well as directly support future robotic and human exploration. We recommend that NASA sustain a vigorous community-based planetary geologic mapping program, including creation of digital (GIS) map products at scales relevant for the identification of new science targets. Higher resolution maps would benefit from the flight of instruments capable of acquiring diverse, high-resolution datasets that afford sub-meter feature identification. NASA should require the inclusion of formal map production as an early planning phase for each new mission, and the production of the necessary digital databases to support this effort. NASA should actively support the continued growth and development of planetary geologic mapping through investments in the mapping community, including mentoring of a diverse community of younger investigators by senior researchers. NASA should fund the production of global geologic maps of all newly explored planetary objects.
\end{abstract}

\title{
1. INTRODUCTION
}

Planetary geologic mapping - that is, mapping of all solid-surface bodies in the Solar System other than Earth - is a remote-sensing-based, fundamental research endeavor that helps to establish the geologic setting of planetary bodies in parallel with other geoscience disciplines using data acquired by spacecraft (i.e., fly-by images, orbiters, and landed spacecraft). Planetary geoscience maps, often products of the National Aeronautics and Space Administration (NASA)/U.S. Geological Survey (USGS) Planetary Geologic Mapping Program, present fundamental syntheses of interpretation of the materials, landforms, structures, and processes that characterize the surface and shallow subsurface of planetary bodies. Beyond producing original and high-impact discoveries [1,2], such maps offer a basis for evaluating thematic research for a given region or body and for global summaries of diverse geologic analyses [3]. Geoscience maps, through incorporation of age constraints derived from stratigraphic relationships and populations of impact craters, serve as a primary means to evaluate the temporal evolution of planetary surfaces.

The information and context provided by planetary geoscience maps promote basic and applied research within and across various planetary geoscience disciplines. In addition, these products provide critical context for programmatic and policy decisions. Since 1961, planetary geoscience maps have been used in nearly every facet of exploration, from landing site characterization for human [4] and robotic [5] missions, to establishing regional and global stratigraphy [6, 7], and assessing the structural geology characteristics of regions of particular scientific value [8].

Geoscience mapping campaigns are sometimes included in mission proposals as anticipated derivative products [9, 10], along with other high-order cartographic data products such as controlled image mosaics and digital terrain models. Planning documents from multiple planetary-focused programs, organizations, and institutions identify geoscience maps as key scientific and technical contributors to planetary exploration [11]. In line with these community uses and priorities, and in cooperation 
with the USGS Astrogeology Science Center (Flagstaff, AZ), NASA has built and maintained a robust infrastructure dedicated not only to producing paper and digital geoscience maps but also to building and disseminating mapping-based resources to the international planetary science community.

NASA has supported the geologic mapping of planetary surfaces since the 1960s, working in conjunction with the USGS through a variety of organizational structures. Planetary geologic mapping has been a fundamental component of NASAQ̂́ exploration of the Solar System and has been applied to all the terrestrial planets, the outer planet satellites, and small bodies (dwarf planets, asteroids and comet nuclei) for which adequate image coverage is available. Mapping scales have typically varied from global (e.g., 1:20,000,000 to $1: 15,000,000$, depending upon the size of the body) to regional (e.g., 1:500,000 to $1: 5,000,000$ ), and increasingly commonly some maps are produced at scales of $1: 18,000$ [8] to $1: 200,000$ [12]. In addition to traditional hard copy map products, modern planetary geoscience maps convey complex observations and interpretations of orbital data through the use of digital products that employ geographic information system (GIS) software and file formats [13]. In the coming decade, with the return to the Moon and the development of increasingly sophisticated spacecraft destined for numerous Solar System destinations (including 16 Psyche, Titan, Europa, and numerous other bodies), the need for enhanced planetary geologic mapping is pressing. Such mapping is a critical activity that underpins and promotes future scientific investigations and has several important modes: 1) global syntheses; 2) as an element of focused research on a specific topic, region, or process; and 3) in support of planning for orbital, robotic, and human missions. These modes are described as follows for different parts of the Solar System.

\section{Why Planetary Mapping?}

NASAQ̂́ Mapping and Planetary Spatial Infrastructure Team (MAPSIT) has identified the need for community access to multiple calibrated and geo-rectified data sets for all Solar System bodies for which adequate data exist. More than ever, standardized geologic (as well as topographic and composition) maps of planetary surfaces are needed to support future NASA missions. Planetary geologic maps are frequently compiled and published at regional to global scales. Though such maps help provide important context for establishing and cross-comparing broadly-occurring units, they cannot directly support future robotic and human exploration of targeted locations. As a result, there is an anticipated demand for geologic maps that use diverse, high-resolution datasets to create geologic maps at both local and regional scales. Local scale maps (for example, geologic maps at 1:5,000 scale, where $1 \mathrm{~mm}$ on the map is equal to $5 \mathrm{~m}$ on the ground) help enable operational decisions at the ground-level for both rover traverses and, potentially, human exploration of the surface.

Because of the diverse science and operational needs of future missions, geologic mapping should proceed with a nested approach, wherein multiple map scales work in tandem to support the fullest range of scientific and technical uses (Table 1). For research projects that generate Scientific Investigation Map (SIM) series products, the USGS is in effect the publication venue for standardized, NASA-funded maps. However, 
a minimum standard is required for the preparation of planetary geologic maps regardless of publication venue, to ensure that these products are interoperable and seamlessly comparable.

Table 1: Correlation between map scale, image resolution, and map utility

\begin{tabular}{|r|r|r|l|}
\hline Map Scale & $\begin{array}{r}1 \mathrm{~mm} \text { of map }= \\
\text { XX m on ground }\end{array}$ & $\begin{array}{r}\text { Image Res. } \\
\text { (m/pixel) }\end{array}$ & Utility \\
\hline $1: 500$ & $0.5 \mathrm{~m}$ & 0.25 & Identification of science targets and hazards \\
\hline $1: 5,000$ & $5 \mathrm{~m}$ & 2.5 & Construction, resource assessment, traversing \\
\hline $1: 50,000$ & $50 \mathrm{~m}$ & 25 & Long-distance traversing, resource assessment \\
\hline $1: 500,000$ & $500 \mathrm{~m}$ & 250 & Link between local and regional/global units \\
\hline
\end{tabular}

Lacking in the previous mapping programs supported by NASAôs ROSES initiatives has been the extensive incorporation of other data sets. Laser altimeter instruments such as the Mars Orbiter Laser Altimeter (MOLA) and its lunar counterpart (LOLA) have produced unprecedented digital topographic data that can be incorporated into maps for science analysis (e.g., unit thickness, volumes, slope, aspect, etc.) as well as terrain hazards (meter-scale roughness, slopes, and other lander- and rover-relevant hazards). Thermal infrared (TIR) data from the DIVINER instrument have been used to determine boulder distributions on the Moon [14], and geochemical data for the Martian surface have been obtained via the CRISM spectrometer [15]. Radar data for Titan and gamma ray spectral data for the Moon and Mars should all be folded into next-generation geologic maps of these bodies. Other data sets, such as spectral data for Mercury or radar emissivity data for Venus, could also be utilized. Fortunately, with the advent of the production of digital versions of the maps through the USGS SIM products, such diverse data can be accessed in the same coordinate system as visible-wavelength image data.

\section{Return to the Moon}

The NASA Constellation Program Office and the 2009 Lunar Exploration Analysis Group (LEAG) workshop on sustaining lunar exploration, together with the lunar science community, identified 50 priority areas and developed a list of $>16,000$ potential areas of interest on the Moon. Thereafter, the Lunar Science for Landed Missions workshop held in January 2018 defined a set of targets that near-term landed missions could visit for scientific exploration. Targets include candidate sites for the study of lunar volatiles, pyroclastic (i.e., volcanic) deposits, and crustal geochemistry. Many targets have been imaged at a scale of a meter or better by the Lunar Reconnaissance Orbiter (LRO) Camera (LROC). These images reveal a Moon with a geologically complex, scientifically compelling, and far more varied surface than might be expected from the limited number of sites visited by previous missions.

The next human and robotic landings on the Moon will likely target the south polar region, with investigation of ices on the floors of permanently shadowed craters one of the highest priority objectives. High-value science may be conducted not only at the lunar poles but also at lower latitudes on the nearside and farside. For all such studies, LROC Narrow Angle Camera (NAC) images and other data sets (e.g., LOLA altimetry) 
support larger-scale mapping to understand study areas via unit descriptions, hazard mapping, and resource concentrations. Many parts of the Moon have only been formally mapped at 1:5,000,000 scale prior to the availability of modern remote-sensing data [16]. Further, no maps currently exist at the required scale(s) to navigate humans and robots on the lunar surface, nor to place localized observations into a regional context to understand a given regionô overall geologic history. It is therefore concluded that a new lunar mapping program is vital, and recommend its establishment to both identify the most important scientific objectives and provide support for mission planning for future lunar polar exploration.

Early lunar South Pole maps were produced at a scale of 1:5,000,000 by $[16,17]$. A mosaic of the South Pole was prepared by the USGS using LRO Wide Angle Camera (WAC) images at a scale of $\sim 1: 6,000,000$. Map products at 1:300,000 derived from LRO data sets are available at www.lpi.usra.edu/lunar/lunar-south-pole-atlas/. However, much more detailed maps will be needed prior to any future landings. A few years after the Apollo landings, the Defense Mapping Agency prepared maps of traverses at the Apollo landing site at a scale of 1:25,000, which approximates the most likely scale for maps for future exploration. Speyerer et al. [18] developed a traverse planning tool for future lunar polar missions based on LOLA data. However, as the tool used topographic data rather than image data, the map derived by [18] for the potential traverse does not convey what astronauts would see on the ground.

The polar regions of the Moon present unique challenges never faced by planetary mappers. The pervasive long shadows because of low solar incidence angles, lighting from multiple azimuth directions providing rare glimpses of the surface, and the enhancement of morphological features perpendicular to the lighting direction conspire to make map production a very different task from that at lower latitudes and less challenging illumination conditions.

\section{Mars Science}

The use of Mars Reconnaissance Orbiter (MRO) Context Camera (CTX) data as a primary image base, complemented by the suite of other datasets for Mars (including high-resolution topography) represents an incredibly valuable resource for larger-scale geologic mapping of Mars in many areas of the planet. For example, maps of candidate landing sites for the Mars 2020 Perseverance rover were central to the selection of the ultimate landing site at Jezero Crater [19]. Identifying morphological evidence for standing bodies of water and/or fluvial activity (e.g., deltaic deposits, shorelines, etc.), as well as assessing the probability of sample collection, both relied on the production of high-resolution geologic and morphologic maps for this site. Mapping of this type this should continue, given its demonstrated importance in supporting landing site selection studies.

The identification of site-specific geologic context, and potential access to resources (e.g., ground ice), using diverse multi-spectral images and other data sets, will be key to establishing a sustainable human presence on Mars. Such data will also help characterize the activities that must be carried out on the surface. Crewed missions to 
Mars will require the same types of geologic spatial information as lunar exploration. This information includes, but is certainly not limited to, the distribution of slopes at various scale-lengths, the abundance and size distribution of rocks, the overburden depth of targeted mineral types, and the concentration and distribution of these minerals - all of which can be synthesized into useful geologic map products.

\section{Giant Planet Satellites}

The in situ exploration of the surface of Europa by a landed spacecraft would be dramatically enhanced by the production of geologic map products from Europa Clipper and Galileo images. Similarly, the regional context of Dragonfly data for the surface of Titan from that missionôs planned multiple sorties [20] will rely on context data from the Cassini radar to identify safe landing sites as well as scientifically interesting targets. New types of maps involving a temporal component may also be required for missions to Jupiterô moon Io, where active volcanism is sufficiently dynamic that the landscape can change between missions (e.g., Galileo vs. Juno, and Europa Clipper vs. any potential future mission to Io). Orbital and surface investigations that are early in the mission development phase would benefit greatly from geologic mapping and global syntheses. For areas with higher resolution data, additional detailed regional or local mapping should be conducted.

\section{Data Sets}

Most planetary geologic maps currently employ visible image data as the primary data set. Compositional and thermophysical data, as well as topographic, subsurface (via radar sounding), and geophysical (e.g., gravity) observations, are increasingly used to characterize a planetary surface and subsurface to maximize science returns [21], and will only become more important to the creation of new geologic maps. The integration of multiple, varied data sets, such as those now available through the NASA Planetary Data System (PDS) Geosciences and Imaging Nodes, for systematic documentation of planetary surfaces has the potential to further enhance the value of planetary geologic maps. This integration will probably require additional time commitments and/or assembling larger mapping teams than those utilized in traditional mapping that is based only on visible imaging data sets.

\section{Recommendations}

a. NASA should continue to fund planetary geoscience mapping as a core investigative tool for exploring rocky and icy bodies in the Solar System. Important types of mapping studies include: a) topically-driven local to regional scale mapping to address unresolved issues regarding surface processes and/or geologic history; $b$ ) regional to global syntheses as part of maturation of geologic understanding of an object; c) mapping that integrates multiple, diverse datasets (e.g., imaging, topographic, compositional); and d) global geologic maps of newly imaged planetary objects to synthesize results of flyby and orbital missions.

b. Based upon the demonstrated critical value to NASA missions of geologic mapping, ranging from site selection of the Apollo landing sites to the operation of the Mars rovers (Spirit, Opportunity, Curiosity and, soon, Perseverance), we strongly 
recommend that NASA sustain a vigorous community-based planetary geologic mapping program that includes creation of digital (GIS) map products at scales relevant for the identification of new science targets, access to future in situ resources, and the optimal selection of landing sites on the Moon, Mars, Europa, Titan, and elsewhere.

c. Complementing these maps of new targets, additional high-resolution maps should be supported that explore science topics at a finer scale with multiple data sets. The higher resolution maps would benefit from the widespread flight of instruments capable of acquiring high-resolution images that allow identification of sub-meter scale geologic features and unit relationships, particularly if the instruments include stereo-imaging capability to produce digital elevation models with horizontal resolution of a few meters per pixel.

d. Experience has shown that planetary geoscience maps may take years to advance from the proposal stage to final publication as a USGS map. This is a fundamental aspect of the multiple inter-related aspects of map creation, including the production of the digital linework, the description of map units, and the extensive scientific and cartographic review process that results in a standardized map. Yet it is possible that several dozen new maps at a scale of 1:20,000 to 1:250,000 may be needed to support future lunar missions. To sustain such an effort, NASA should require the inclusion of formal map production at an early planning phase for each new mission, and the production of the necessary digital data bases to support this effort.

e. To meet the need for a new generation of planetary geoscience maps, NASA should actively support the continued growth and development of the planetary geologic mapping community, including mentoring of younger investigators by senior researchers, and through the hosting of topical workshops focused on mapping techniques.

\section{References}

[1] Tanaka, K.L. (2005). Geology and insolation-driven climatic history of Amazonian north polar materials on Mars. Nature 437, 991 ï 994. [2] Griffith, C.A. et al. (2019). A corridor of exposed ice-rich bedrock across Titanôs tropical region. Nature Astronomy 3, 642 ï 648. [3] Skinner, J.A., Fortezzo, A.E., Gaither, C.M., Hare, T., Hunter, M.A., Buban, H. (2019). Planetary geologic mapping $̈$ I Program status and future needs. USGS Open File Report 2019-1012, 40 pp. [4] Grolier, M. J. (1970). Geologic Map of the Apollo Landing Site 2 (Apollo 11): Part of Sabine D region, Southwestern Mare Tranquilitatis, USGS Miscellaneous Map I-619, 1:25,000 scale. [5] Anderson, R.B., Bell, J.F. (2010). Geologic mapping and characterization of Gale Crater and implications for its potential as a Mars Science Laboratory landing site, Mars Journal, 5, 76-128, doi:10.1555/mars.2010.0004. [6] Tanaka, K.L. and 8 others (2014). Geologic Map of Mars. USGS Scientific Investigations Map 3292. [7] White, O.L. and 23 others (2017). Geological mapping of Sputnik Planitia on Pluto. Icarus 287, 261 ï 286. [8] Okubo, C.H., Gaither, T.A. (2017). Bedrock and structural geologic maps of Eastern Candor Sulci, Western Ceti Mensa, and Southeastern Ceti Mensa, Candor Chasma, Valles 
Marineris Region of Mars. U.S. Geological Survey Scientific Investigations Map 3359. [9] Williams, D.A., Yinst, R.A., Garry, W.B. (2014). Introduction: The geologic mapping of Vesta. Icarus 244, 1 ï 12. [10] Williams, D.A, Buckowski, D.L., Mest, S.C., Scully, J.E.C., Platz, T., Kneissl, T. (2018). Introduction: The geologic mapping of Ceres. Icarus 316, 1 ï 13. [11] Hendrix, A.R. and 27 others (2019). The NASA Roadmap to Ocean Worlds. Astrobiology 19(1), doi:10.1089/ast.2018.1955. [12] Mouginis-Mark, P.J. (2015). Geologic map of Tooting crater, Amazonis Planitia region of Mars. U.S. Geological Survey Scientific Investigations Map 3297. [13] Hare, T.M., Kirk, R.L., Skinner, J.A., Tanaka, K.L. (2009). Chapter 60: Extraterrestrial GIS, in Madden, M., Ed, Manual of Geographic Information Systems: Bethesda, American Society for Photogrammetry Remote Sensing, p. 1199 ï 1219. [14] Bandfield, J.L., Ghent, R.R., Vasavada, A.R., Paige, D.A., Robinson, M.S. (2011). Lunar surface rock abundance and regolith fines temperatures derived from LRO Diviner Radiometric data. J. Geophys. Res. 116 (E12), doi:10.1029/2011JE003866. [15] Pelkey, S.M., and 11 others (2007). CRISM multispectral summary products: Parameterizing mineral diversity on Mars from reflectance. J. Geophys. Res. 112 (E8), doi:10.1029/2006JE002831. [16] Wilhelms, D.E., Howard, K.A., Wilshire, H.G. (1979). Geologic map of the South Side of the Moon. USGS Miscellaneous Map I-1162. [17] Aeronautical Charts and Information Service (1970). Lunar Polar Chart (LMP-3). [18] Speyerer, E.J., Lawrence, S.J., Stopar, J.D., Glaser, P., Robinson, M.S., Jolliff, B.L. (2016). Optimized traverse planning for future polar prospectors based on lunar topography. Icarus 272, 337 ï 345. [19] Grant, J.A., Golombek, M.P., Wilson, S.A., Farley, K.A., Williford, K.H., Chen, A. (2018). The science process for selecting the landing site for the 2020 Mars Rover. Planet Space Sci. 164, 106 ï 126. [20] Lorenz, R.D. and 17 others (2018). Dragonfly: A rotorcraft lander concept for scientific exploration at Titan. APL Tech. Digest 34 (3), www.jhuapl.edu/techdigest. [21] Ono, M., Rothrock, B., Almeida, Ansar, A., Otero, R., Huertas, A., Heverly, M. (2016). Data-driven surface traversability analysis for Mars 2020 landing site selection. IEEE Aerospace Conf., doi: 10.1109/AERO.2016.7500597. 